[Aus dem Hygienischen Institut der Universität Berlin

und dem Hygienischen Institut der Universität Greifswald.]

\title{
Über die säurefesten Trompetenbazillen.
}

I. Mitteilung.

Von

K. E. F. Schmitz,

z. Zt. stellvertretender Direktor des Hygien. Instituts in Greifswald.

(Hisrxu Taf. VIII u. IX.)

\section{Geschichtliches.}

Gelegentlich der Untersuchung eines tuberkulösen Trompeters kamen im Jahre 1909 Jacobitz und Kayser ${ }^{2}$ auf den Gedanken, die von diesem Musiker benutzten Trompeten auf das Vorhandensein von Tuberkelbazillen zu untersuchen, um eine auf diesem Wege ja mōgliche Ansteckung dritter, Personen zu verhindern. Sie machten bei dieser Gelegenheit die auBerordentlich interessante Entdeckung, daB sich in fast allen untersuchten Trompeten säurefeste Bazillen in ganz ungeheuren Mengen fanden.

Gerade dieser Umstand aber, daB sich diese säurefesten Bazillen in so groBen Mengen hier vorfanden, verursachte, daB die genannten Autoren Kontrolluntersuchungen derart anstellten, $\mathrm{daB}$ sie erstens eine gröBere Anzahl ron Trompeten auf das Vorhandensein dieser Säurefesten untersuchten, und ferner, daB sie mit diesem Trompetenschleim Meerschweinchenimpfungen vornahmen, um festzustellen, ob es sich wirklich um Tuberkelbazillen handelte.

Die Untersuchung von 29 Blechinstrumenten ergab nun das Resultat, dab sich in 27 von ihnen, also fast allen, säurefeste Stäbchen

1 Münchener med. Wochenschrift. 1910. S. 1175. 
nachweisen ließen. Die mit dem Schleim von 22 Instrumenten gespritzten Meerschweinchen zeigten aber bis auf ein einziges keine tuberkulöse Erkrankung, und dieses eine war mit Schleim gespritzt worden aus einer Trompete, die von dem eingangs erwähnten Musiker geblasen worden war.

Aus diesen Befunden schlossen die Autoren, daB es sich um einen Mikroorganismus handeln müsse, der auf jedem Fall nicht mit dem Tuberkelbacillus identisch ist. Genaueres über die Art der beschriebenen Bazillen konnten Jacobitz und Kayser nicht feststellen, da es ihnen trotz mehrfacher Bemühungen nicht gelang, sie zur Kultur zu bringen. Nur einmal ging eine gelbe Kolonie auf, aus der säurefeste Bazillen gewonnen werden konnten. Auch über die Herkunft der Bazillen konnten sie nichts feststellen.

Diese säurefesten Bazillen fanden sich am reichlichsten in Blechinstrumenten und zwar Trompeten, Waldhörnern, Tenorhörnern, Posaunen, Tuben usw. Auch in Holzinstrumenten konnten sie, wenn auch bedeutend spärlicher, nachgewiesen werden, aber immer nur dort, wo sich Feuchtigkeit vorfand.

Es ist noch zu bemerken, daB es Jacobitz und Kayser mehrmals gelang, solche säurefesten Bazillen in dem Mund- und Rachenschleim von Trompetern nachzuweisen, eine für die Tuberkulosediagnose auBerordentlich wichtige Feststellung.

Obwohl, wie Jacobitz und Kayser festgestellt haben, diese Bazillen in fast allen Trompeten in großen Massen nachzuweisen sind, dauerte es doch geraume Zeit, bis die Forschungen über diese eigentümlichen, aber für die Tuberkulosediagnose durchaus nicht gleichgültigen Bazillen von Heymann und Seidel wieder aufgenommen wurden. Auch sie konnten in allen untersuchten Trompeten dieselben Stäbchen nachweisen. Im Gegensatz zu Jacobitz und Kayser konnten sie aber beobachten, daB diese Stäbchen, wenn sie nur in genügender Menge eingespritzt werden, im Tierversuch durchaus nicht vollkommen avirulent sind. ${ }^{1}$ Sie beobachteten mächtige Schwellungen und Vermehrung der Bazillen in den nächstgelegenen Drüsen, die öfters bis Kirschgröße anschwollen und dann entweder aufbrachen und einen dünn]äsigen Eiter von sich gaben oder von selbst wieder zurückgingen.

Auf Anregung von Hrn. Geheimrat Flügge übernahm ich sodann im Jahre 1913 die weitere Bearbeitung des Themas. Meine Bemühungen erstreckten sich zunächst auf die Feststellung, wo diese Säurefesten her-

1 Centralblatt für Bakteriologie. Ref. I. Abt. 1913. Bd. LVII. Bericht über die 7. Tagung der freien Vereinigung für Mikrobiologie. S. 293. 
kämen, und sodann auf ihre Kultivierung. Fernerhin sollte dann die Morphologie und Pathogenität der Bazillen nach ihrer Kultivierung genauer festgestellt werden.

\section{Verbreitung und Herkunft der Trompetenbazillen.}

Bei meinen Untersuchungen, sowohl in Berlin wie in Greifswald, konnte ich feststellen, daB in allen Blechinstrumenten die säurefesten „Trompetenbazillen" wie sie von Hejmann kurz benannt worden waren, zu finden sind, ganz gleichgültig ob die Instrumente von Militär- oder Zivilmusikern stammten. Wie ich weiterhin feststellen konnte, scheinen sie aber in ungebrauchten Instrumenten zu fehlen, wenigstens vermochte ich sie in zwei neuen Trompeten und einer Mundharmonika nicht zu finden.

Der Sitz der Bazillen in den Trompeten ist auch gerade da, wo sich am meistem Schleim und Schmutz anzusiedeln pflegt, also z. B. in den Biegungen der Röhren, ferner sehr reichlich an den Ventilen, besonders an deren konkaven Flächen. Streift man den dort sitzenden graugrünlichen Schleim ab und färbt ohne weiteres nach Ziehl, so, findet man sie gewöhnlich im ersten Präparat schon in großen Mengen, oft zu Klumpen vereinigt (s. Fig. 3, Taf. IX). Da die Bazillen in ungebrauchten Trompeten zu fehlen scheinen, so ist es höchst wahrscheinlich, daB dieselben durch den Gebrauch hineingeraten. I Leider war es jedoch nicht möglich festzustellen, in welcher Weise dies zu geschehen ptlegt. Immerhin konnte ich durch Befragen der Musiker folgendes feststellen:

1. Sie finden sich auch in Trompeten, die immer nur von demselben Musiker geblasen wurden.

2. Die Reinigung der Instrumente wurde von fast allen so angegeben, daB die Trompete mit dem Mundstück von Zeit zu Zeit an einen Wasserhahn gehalten wird, und daB man dann einen scharfen Wasserstrahl durch die Trompete durchschieBen läBt. Es wäre möglich, daB auf diese Weise säurefeste Bazillen, die sich ja bekanntlich auBerordentlich häufig in Wasserhähnen finden, in die Trompete hinein verschleppt würden.

3. Gaben mir viele der Musiker an, dab sie öfters die Ventile der Trompeten mit verschiedenen Fetten, Vaseline, manchmal auch Butter einschmieren, um sie besser gleiten zu lassen. Es wäre auch möglich, $\mathrm{daB}$ auf diesem Wege die säurefesten Bazillen in die Trompete hineingelangen.

DaB etwa die Trompetenbazillen aus dem Körper der Trompeter selbst in die Trompete gelangen, erscheint mir wenig wahrscheinlich, da es mir im Gegensatz zu Jacobitz und Kayser kein einziges Mal gelungen ist, im Auswurf oder in Rachenabstrichen der Trompeter dieselben săurefesten Stäbchen nachzuweisen, selbst wenn sich in den ihnen gehörigen Trom- 
peten, auf denen sie manchmal täglich stundenlang musizierten, die Bazillen in ungeheuren Mengen fanden. Im gleichen Sinne deute ich auch einen allerdings nur negativ ausgefallenen Versuch. Obwohl ich wochenlang täglich selbst auf einer vorher untersuchten und rein befundenen neuen Trompete geblasen habe, ließen sich nach dieser Zeit in derselben.keine säurefesten Bazillen nachweisen. Die Trompete war weder geölt, noch an der Wasserleitung gereinigt worden.

Allerdings ist bei diesem Versuch der Einwand berechtigt, daB es vielleicht notwendig ist, ein Instrument täglich mehrere Stunden zu benutzen, um die Bazillen zur Wucherung zu bringen. Solches war mir jedoch nicht möglich.

\section{Reinkultur.}

Die Kultur der săurefesten Trompetenbazillen war den Voruntersuchern im wesentlichen nicht gelungen. Wie oben schon erwähnt, hatten Jac obitz und Kayser einmal eine gelbe Kolonie entdeckt. Ferner beobachtete Heymann in mit Messingspänen versetzten Nährböden zweifellose Vermehrung, aber zu einer gesicherten Reinkultur war man noch nicht gelangt. Dieselbe gelang uns jedoch außerordentlich rasch mit Verwendung des Antiforminverfahrens, und zwar wurde so vorgegangen, da $B$ der Trompetenschleim mit 10 bis 20 prozentigme Antiformin versetzt und eine Stunde im Brutschrank aufbewahrt wurde. Dann wurde zentrifugiert, der Bodensatz mehrmals mit sterilem Wasser gewaschen. SchlieBlich wurde der Bodensatz auf Lu ben a us Glyzerin-Eier-Nährboden ausgestrichen, und sowohl bei $37^{\circ}$ als auch bei Zimmertemperatur das Wachstum beobachtet. Einmal gelang es auch aus einem Knoten, der sich bei einer subkutanen Impfung beim Meerschweinchen gebildet hatte, die Bazillen ohne weiteres herauszuzüchten.

Für die erste Züchtung eignete sich der Lubenausche Eier-Nährboden weitaus am besten, nächst ihm zeigten sich auch Glyzerinkartoffeln brauchbar. Ist es einmal zu einem ordentlichen Wachstum gekommen, so wuchert der Bacillus auBerordentlich - uppig auf allen glyzerinhaltigen für die Tuberkelbazillen gebräuchlichen Nährböden. LäBt man das Glyzerin fort, so tritt kein Wachstum auf. Er wächst bei $37^{\circ}$ rascher als bei Zimmertemperatur.

Bei der ersten Herauszüchtung dauert gewöhnlich das Aufkommen der Kolonien geraume Zeit bis zu 4 und 6 Wochen. Ist die Kultur aber erst einmal ordentlich im Schwunge, so vermag der Bacillus viel rascher zu wachsen als der Tuberkelbacillus. Schon nach 3 bis 4 Tagen kann man dann deutliches Wachstum beobachten, und nach 1 bis 2 Wochen kann bereits eine gute Kultur erzielt werden. Das Aussehen der Trompeten- 
bazillenkulturen erinnert außerordentlich stark an die Tuberkelbazillenkulturen, dicke bröckelige und höckerige Beläge, gewöhnlich etwas weiBlich, manchmal, besonders auf Glyzerinkartoffeln etwas gelblich gefärbt. Junge Kulturen pflegen ziemlich feucht zu sein; die älteren sind trockener und werden dadurch den Tuberkelbazillenkulturen noch ähnlicher. Hanchmal bilden sich bei diesen älteren Kulturen auf dem Rasen noch eigentümliche Innöpfe von etwas über Stecknadelkopfgröße.

Auch auf der Oberfläche von Glyzerinbouillon sind sie ziemlich leicht zu züchten. Auch hier erinnern sie stark an das Aussehen der Tuberkelbazillen. Doch kounte beobachtet werden, daß die Bewachsung der Oberfläche verschieden erfolgt, je nachdem man ein dickes Stück oder nur ein ganz feines Stück auf die Oberfläche impft. Im ersteren Falle wächst die Oberfläche weniger raseh zu. Es bilden sich lauter dicke Bröckel, während im zweiten Falle eine rasche Verbreitung über die ganze Oberflāche erfolgt. Die so gebildete Haut verdickt sich dann zu ungleichmäBigen, nußkernartig gezackten und gekrümmten Wülsten (s. Fig. 1, Taf. VIII). Ist die ganze Oberfläche der Bouillon bewachsen, so vermag sich das Häutchen noch ungefähr $1 / 8 \mathrm{~cm}$ an der Glaswand hinaufzuschieben. Die Bouillon wird nicht getrübt. Etwa herabgefallene Stücke wachsen unten zu dicken Klumpen aus.

Das Zuwachsen der Oberfläche geschieht sehr rasch, sobald die Bazillen sich einmal an diese Wachstumsform gewöhnt haben. Bereits in 5 bis 6 Tagen kann die Oberfläche eines kleinen Kölbchens mit einer feinen Decke überzogen sein. Diese starke Wachstumsenergie erklärt es, daB die Bouillonkulturen nicht so leicht wie Tuberkelbazillen-Bouillonkulturen durch Infektion mit anderen Keimen vernichtet werden. Wenn solche Luftinfektion eintritt, so wachsen doch die Trompetenbazillen zusammen mit den Liuftkeimen weiter, in der Weise, daB sich die Trompetenbazillen ruhig an der Oberfläche weiter entwickeln, während die Kokken die übrige Bouillon durchsetzen. Die Bouillonreinkultur hat einen gewissen feinen an Tuberkulin erinnernden Geruch. Dieser pflegt, falls die eben beschriebene Mischinfektion eingetreten ist, einem eigentümlichen säuerlichen Geruche zu weichen.

Nachdem einmal die Kultur der Trompetenbazillen gesichert war, war es nun ein leichtes, mit derselben die verschiedensten Versuche, besonders im Vergleich mit Tuberkelbazillen, anzustellen.

$\mathrm{DaB}$ der Trompetenbacillus auch antiforminfest ist, geht schon aus dem .oben erwähnten Umstande herror, daB es gerade mit der Antiforminmethode gelang, den Trompetenbacillus rein zu züchten. Einmal gelang sogar die Kultur, nachdem das Ausgangsmaterial in unverdünntem Antiformin eine Stunde aufbewahrt worden war. 
In der Morphologie ähnelt der Trompetenbacillus ubsolut dem Tuberkelbacillus. Das einzelne Stäbchen ist von Tuberkelbazillen überhaupt nicht zu unterseheiden.

Die Anordnung im mikroskopischen Bild der Reinkultur zeigt zwar nicht so typisch das zopfförmige Aussehen wie beim Tuberkelbacillus, doch konnten Anklänge hieran auch öfters beobachtet werden. Jedenfalls hat der Bacillus Neigung zur Gruppenlagerung, und ist das homogene Verreiben auf dem Deckgläschen ganz wie beim Tuberkelbacillus erschwert. Gerade wie der Tuberkelbacillus zeigen die Trompetenbazillen auch das merkwürdige Phänomen, daß sie beim Abbrennen von der Platinöse unter starkem Knistern auseinanderspringen.

Die Färbbarkeit ist wenigstens auch bei älteren Kulturen dieselbe wie der Tuberkelbazillen. Ich prüfte die Sãurefestigkeit der Trompetenbazillen in der Weise, dab ich sie mit Tuberkelbazillen, Typus humanus, auf denselben Objekttrāger brachte und vach der Färbung in siedendem Karbolfuchsin den ganzen Objektträger verschieden lange dem Salzsäurealkohol aussetzte. Es ergab sich da kein Unterschied gegenüber den Tuberkelbazillen, selbst bei einstündiger Einwirkung. Es ist nur zu bemerken, daB junge Trompetenbazillen, $d . h$. solche, die noch nicht mindestens 3 Wochen sich in Kultur befinden, das Karbolfuchsin bei der Entfärbung etwas abgeben, so daB eine eigentümlich körnige Färbung resultiert. Der Unterschied ist an den Abbildungen 3 und 4, Taf. IX zu sehen.

SchlieBlich wurde bestimmt, ob die Trompetenbazillen bei ihrem Wachstum auf Glyzerinbouillon in der Lage sind, Säure oder Alkali zu bilden. $\mathrm{Zu}$ diesem. Zwecke wurden zunächst zwei Serien von Glyzerinbouillon-Röhrchen, je genau mit 10 com Flüssigkeit, mit Trompetenbazillen beimpft und durch Titration gegen Phenolphtalein am 1., 2., 4., 6. usw. Tage der Säuregrad der F'lüssigkeit bestimmte. Die Glyzerinbouillon war bei diesem ersten Versuch etwas sauer gewählt worden. Die Titration der unbeimpften Kontrollröhrehen ergab, daB im Mittel ron $10 \mathrm{ccm}$ Bouillon $1.8 \mathrm{~cm} \mathrm{n} / 10 \mathrm{NaOH}$ verbraucht wurde.

\section{Tabelle I.}

Titration der Trompetenbazillen-Bouillonkultur. Säuregrad der Ausgangsbouillon im Mittel $1.8^{\mathrm{ccm}} \mathrm{n} / 10 \mathrm{NaOH}$. Die beimpften Röhrchen (je $10 \mathrm{ccm}$ ) verbrauchten:

$\begin{array}{crl}\text { nach } & 24 & \text { Stunden } \\ " & 2 & \text { Tagen } \\ " & 4 & \\ " & 6 & " \\ " & 10 & \end{array}$

$1.6^{\mathrm{cm}} \mathrm{n} / 10 \mathrm{NaOH}$

$1 \cdot 3 \quad$ "

$1 \cdot 6$

$1 \cdot 95$

$1 \cdot 8$, 


\begin{tabular}{|c|c|c|c|}
\hline nach & 15 Tagen & $1.85^{c}$ & $/ 10 \mathrm{NaOH}$ \\
\hline$n$ & $20 \quad n$ & $1 \cdot 4$ & $r$ \\
\hline$"$ & 25 & 1.95 & $n$ \\
\hline r & 30 & $1 \cdot 9$ & $n$ \\
\hline$n$ & 35 & $2 \cdot 0$ & भ \\
\hline
\end{tabular}

Wiederholung des Versuches mit derselben Bouillon ergab folgendes Resultat: Tabello II.

$\begin{array}{crlll}\text { nach } & 24 \text { Stunden } & 1.6 \mathrm{~cm} & \mathrm{n} / 10 \mathrm{NaOH} \\ n & 2 & 1.8 & \\ " & 4 & 1.75 & \\ " & 6 & 1.5 & \\ " & 15 & 1.5 & 1.7 & \\ " & 20 & 1.75 & " \\ " & 25 & 1.75 & \\ " & 30 & 1.5 & \\ " 60 & 1.8 & \end{array}$

Wenn zur Beimpfung eine bereits etwas saure Bouillon benutzt wird, scheint also im wesentlichen derselbe Aziditätstiter beibehalten zu werden. In den ersten Tagen nach der Beimpfung ist höchstens eine leichte Alkalibildung zu bemerken.

Sodann wurde eine Bouillon, die nicht extra angesäuert war, zu demselben Versuch benutzt.

\section{Tabelle III.}

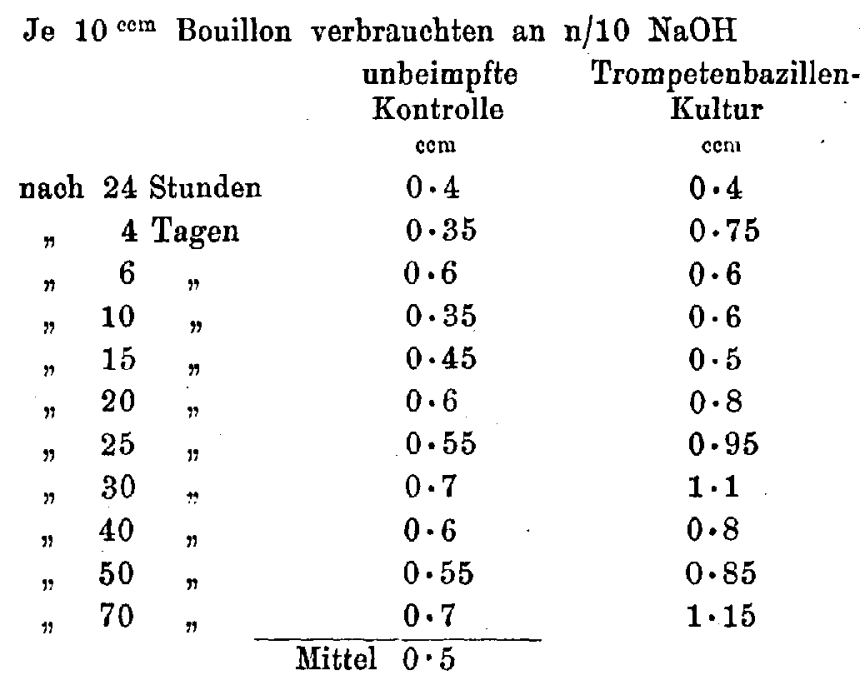


Die Wiederholung desselben Versuches ergab folgendes Resultat:

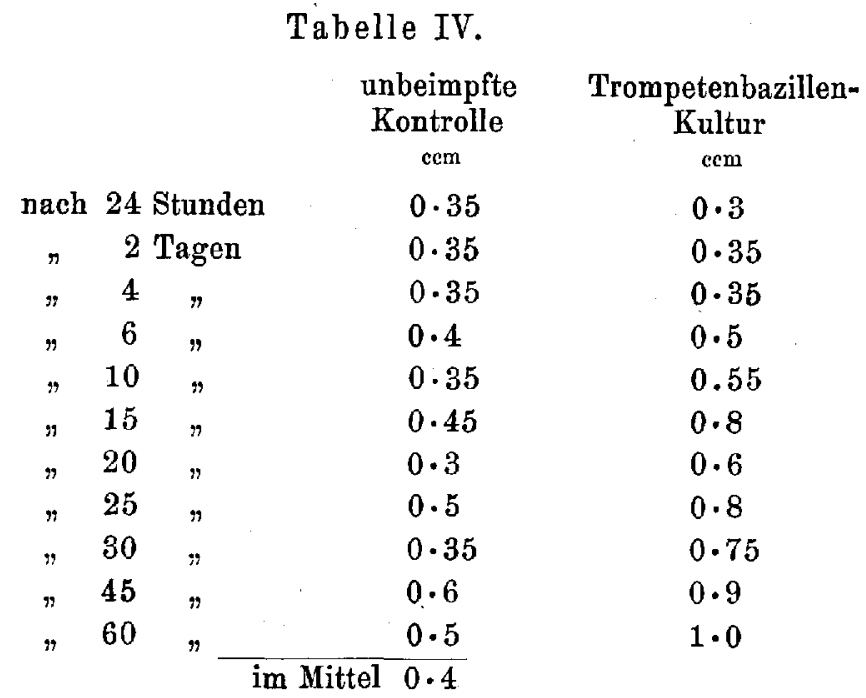

Zuletzt wurden noch fünf verschiedene Bouillonkölbchen mit Trompetenbazillen beimpft, und nach vierwöchentlichem Wachstum die Reaktion titriert. Die Bouillonkölbchen waren vor der Beimpfung so angefertigt worden, daß sie verschiedene Alkalitäts- bzw. Sāuremengen enthielten, und zwar enthielt das erste Kölbchen soviel Alkali, daß $10 \mathrm{cmm}$ seiner Bouillon $1 \mathrm{~cm} \mathrm{n} / 10 \mathrm{HCl}$ zur Neutralisierung verbrauchten. Die Bouillon des zweiten verbrauchte für dieselbe Menge $0.5 \mathrm{~cm} n / 10 \mathrm{HCl}$. Das dritte war gerade - neutral. Das vierte gebrauchte zur Neutralisierung $0.5^{\mathrm{cm}} \mathrm{n} / 10 \mathrm{NaOH}$ und das fünfte und letzte $1.0 \mathrm{n} / 10 \mathrm{NaOH}$.

Nạch vierwöchentlicher Bewachsung fand sich in allen Kölbchen Säure und zwar verbrauchte die Bouillon in dem

\begin{tabular}{|c|c|c|c|c|c|}
\hline Kölbchen & I & $0.5^{\mathrm{cem}}$ & $\mathrm{n} / 10$ & $\mathrm{NaOH}$ pro & $10 \mathrm{~cm}$ \\
\hline$n$ & II & $0 \cdot 6$ & $"$ & $"$ & 10 \\
\hline " & III & $0 \cdot 85$ & ” & 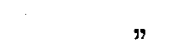 & 10 \\
\hline$n$ & IV & $1 \cdot 0$ & $"$ & $"$ & 10 \\
\hline$n$ & $\mathrm{~V}$ & $1 \cdot 0$ & $n$ & $"$ & 10 \\
\hline
\end{tabular}

Aus dem Ausfall der Versuche 3, 4 und 5 scheint sich also zu ergeben, daB, im Falle die Ausgangsbouillon schwach sauer oder neutral oder gar alkalisch ist, von den Trompetenbazillen Säure gebildet wird, während, wenn die Bouillon bereits einen stärkeren Säuregrad besitzt, stärkere Säurebildung nicht aufzutreten scheint. $\mathrm{Zu}$ dem Versuch 5 ist 
noch zu bemerken, daß das Optimum des Wachstums offenbär bei den Kölbchen mit 0.5 Alkali bis 0.5 Sãure lag. Die beiden Extreme 1.0 Alkali und 1.0 Säure wuchsen nicht so rasch, aber immerhin reichlich.

Wenn wir das Verhalten der Săure- bzw. Alkalibildung mit der gleichen Eigenschaft der Tuberkelbazillen, wie sie ron Theobald Smith zuerst beschrieben worden ist, vergleichen, so können wir einige $\AA$ hhnlichkeit mit dem Verhalten des Tuberkelbacillus, Typus humanus, feststellen. Nach Smith bildet der Tuberkelbacillus Typus bovinus, in einer Glyzerinbouillon, die eine Anfangssäure von ungefähr 2 Prozent enthält, dauernd Alkali, so daB nach einigen Wochen die Reaktion der Bouillon deutlich alkalisch wird. Anders der Typus humanus. Derselbe bildet auch im Anfang etwas Alkali, jedoch nur wenig im Vergleich zum Typus bovinus. Vor allen Dingen wird die Reaktion niemals alkalisch, sondern nach etwa 25 Tagen beginnt eine steigende Säurebildung, die sich gewöhnlich bis zu dem alten Säureniveau erhebt, manchmal dasselbe noch überschreitet. Åhnlich war das Verhalten unserer Trompetenbazillen, nur ist der Verlauf lange nicht so charakteristisch, und ist die Periode der Alkalibildung in der sauren Bouillon sehr viel kürzer (s. Versuch 1 u. 2).

Schon vom 6. Tage ab ist ein deutliches Steigen der Säurebildung zu bemerken. Dieser raschere Ablauf ist vielleicht durch die größere Wachstumsgeschwindigkeit der Trompetenbazillen zu erklären.

Die mit den Trompetenbazillen weiterhin angestellten Versuche, insbesondere die Ergebnisse der Tierimpfungen werden der Gegenstand einer späteren Mitteilung sein, da dieselben bislang noch nicht abgeschlossen sind. 
466 K. E. F. Schmitz: Über die säUrefesten Trompetenbazillen.

\section{Erklärung der Abbildangen.}

(Taf. VIII u. IX.)

(Nach Autochromphotographien vom Verfasser selbst anfgenommen.)

\section{Tafel VIII.}

Fig. 1. Reinkultur von Trompetenbazillen auf der Oberfläche von Glyzerin. bouillon.

Fig. 2. Reinkultur von Trompetenbazillen auf Glyzerinagar.

\section{Tafel IX.}

Fig. 3. Trompetenbazillen im Trompetenschleim. Färbung nach Ziehl. Vergr. etwa 1000 fach.

Fig. 4. Trompetenbazillen aus junger Kultur. Körnige Struktur. Vergr. etwa 1000 fach.

Fig. 5. Trompetenbazillen aus älterer Kultur. Ohne Körnelung. Dieselbe Vergrößerung. 
Zeitschrift für Hygiene. Bd. LXXX.

Tafel VIII.

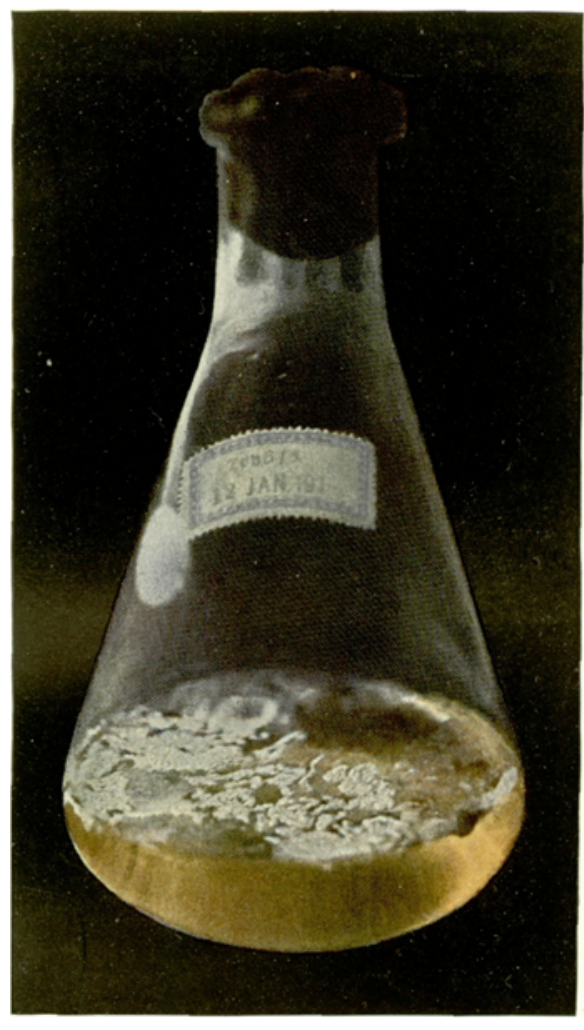

Fig. 1.

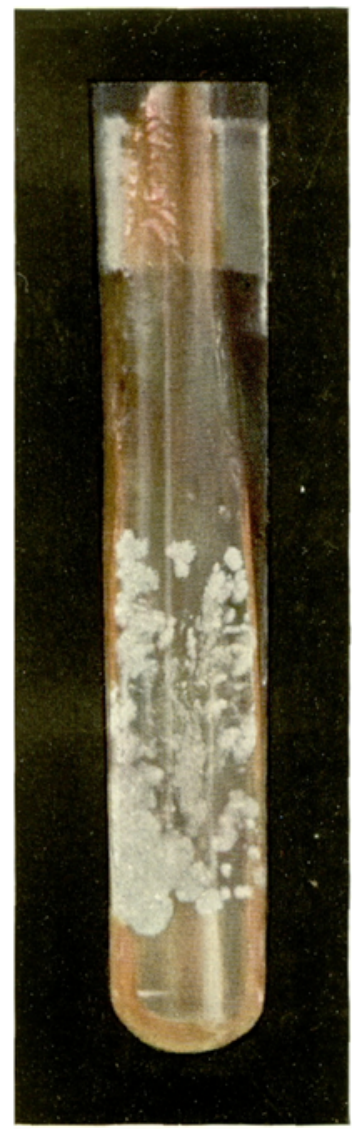

Fig. 2. 
Zeitschrift für Hygiene. Bd. LXXX.

Tafel IX.

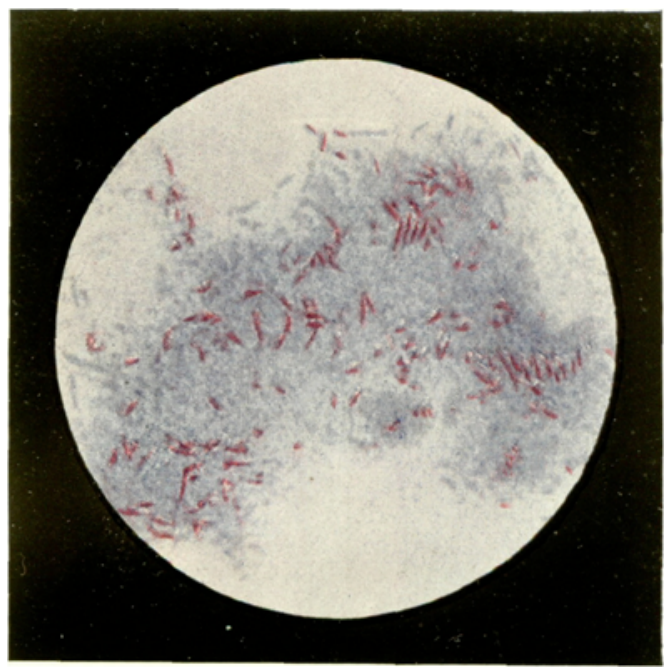

Fig. 3.

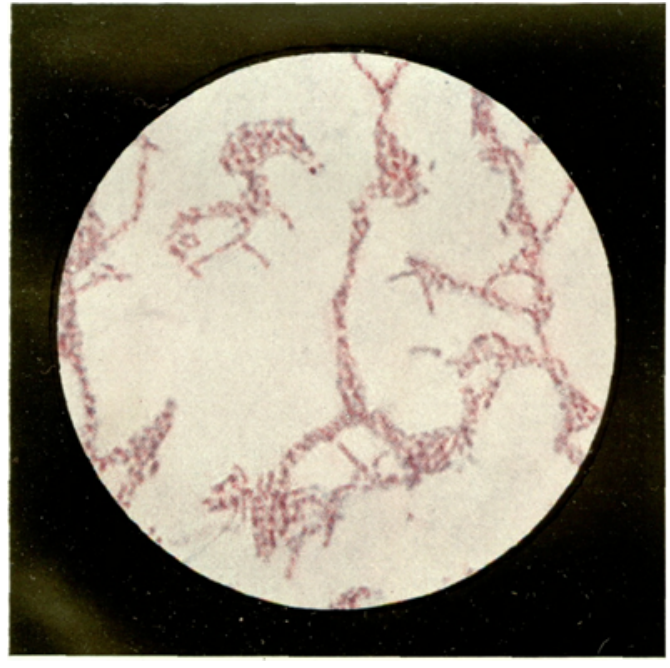

Fig. 4.

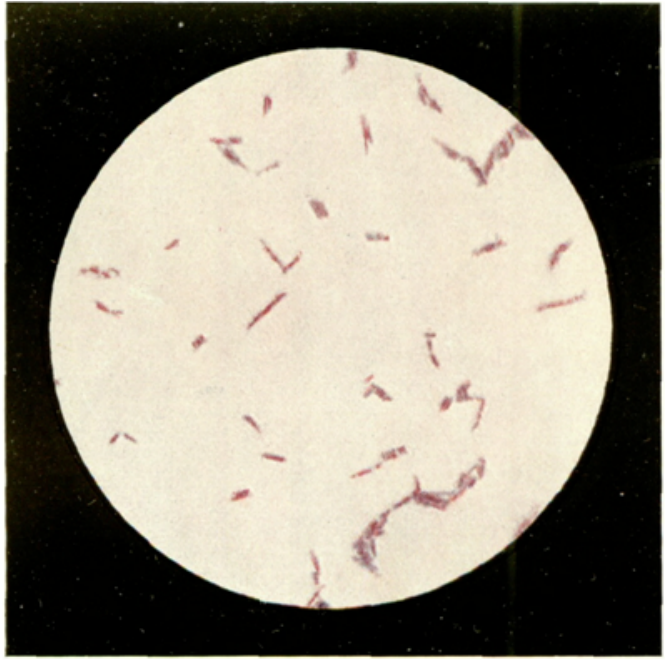

Fig. 5. 